\title{
Letters to the
}

\section{Editor}

\section{Reasons for inability of clopidogrel to inhibit platelet aggregation early after coronary artery bypass surgery To the Editor:}

Clopidogrel administration for platelet inhibition after coronary artery surgery and interventional cardiology is currently a topic of much-deserved attention and debate. I read with interest the recent article in the Journal by Lim and colleagues. ${ }^{1}$ On the basis of the results of an interim analysis of their prospective, double-blind, randomized, controlled trial to compare the efficacy of clopidogrel and aspirin as antiplatelet agents, Lim and colleagues conclude that clopidogrel, unlike aspirin, did not inhibit platelet aggregation in the first 5 postoperative days and therefore should not be used as a sole antiplatelet agent early after coronary surgery. According to the authors, the ineffectiveness of clopidogrel was an "unexpected" finding. However, plenty of evidence is available from published studies to suggest a logical explanation for this unexpected finding.

Clopidogrel is a pro-drug, which is converted to an active, unstable drug by cytochrome P450 (CYP). The active drug irreversibly blocks 1 specific platelet: adenosine 5'-diphosphate receptor (P2Y12). It was recently suggested that the most abundant human CYP isoform, 3A4, activates clopidogrel. $^{2}$ Certain lipophilic statins (ie, simvastatin, atorvastatin, and lovastatin), which are substrates of the CYP3A4 isoform, competitively inhibit the metabolic activation of clopidogrel. As a result, the relative clopidogrel-induced platelet inhibition is diminished, especially if administered in a low dose. ${ }^{3}$ Furthermore, clopidogrel administration results in interindividual variability in platelet inhibition. This variable platelet inhibition response to clopidogrel has been recognized by all who have tested clopidogrel efficacy by platelet aggregometry and correlates with CYP3A4 metabolic activity. ${ }^{4}$ In addition, it is extremely important to remember that platelet inhibition by clopidogrel is dose-related up to a dose of $400 \mathrm{mg}$ and that inhibition at a higher dose remains stable from 2 to 72 hours. $^{5}$

A closer look at the study by Lim and colleagues ${ }^{1}$ clearly reveals that all of these confounding factors are present and that they influence the results of their interim analysis. In my opinion, the conclusion of this study would be different if a larger dose of clopidogrel were used and nonresponders $(\approx 10 \%)$ and low responders $(\approx 20 \%)$, as well as those on statins, were excluded.

Shahzad G. Raja, MRCS Department of Cardiac Surgery Royal Hospital for Sick Children Yorkhill NHS Trust Glasgow G3 8SJ United Kingdom

\section{References}

1. Lim E, Cornelissen J, Routledge T, Kirtland S, Charman SC, Bellm S, et al. Clopidogrel did not inhibit platelet function early after coronary bypass surgery: a prospective randomized trial. J Thorac Cardiovasc Surg. 2004;128:432-5.

2. Clarke TA, Waskell LA. Clopidogrel is metabolized by human cytochrome P450 3A and inhibited by atorvastatin. Drug Metab Dispos. 2003;31:53-9.

3. Neubauer H, Gunesdogan B, Hanefeld C, Spiecker M, Mugge A. Lipophilic statins interfere with the inhibitory effects of clopidogrel on platelet function-a flow cytometry study. Eur Heart J. 2003;24:1744-9.

4. Lau WC, Gurbel PA, Watkins PB, Neer CJ, Hopp AS, Carville DG, et al. Contribution of hepatic cytochrome P450 3A4 metabolic activity to the phenomenon of clopidogrel resistance. Circulation. 2004;109:166-71.

5. Thebault JJ, Kieffer G, Cariou R. Single-dose pharmacodynamics of clopidogrel. Semin Thromb Hemost. 1999;25(Suppl 2):3-8.

doi:10.1016/j.jtcvs.2004.09.027

\section{Reply to the Editor:}

The opinion on the interaction between statins and clopidogrel is still subject to fierce debate. ${ }^{1,2}$ However, excluding sub- 